\title{
In Vitro Competitive Metabolism Study of Olmesartan Medoxomil in Rat Liver S9 Fractions Using LC/MS
}

\author{
Muruganathan Gandhimathi, Rahul Baghla, Sivalingam Subramanian, Thengungal Kochupapy Ravi
}

Department of Pharmaceutical Analysis, College of Pharmacy, Sri Ramakrishna Institute of Paramedical Sciences, Coimbatore, India. Email: gands72@yahoo.co.in

Received January $12^{\text {th }}, 2011$; revised April $16^{\text {th }}, 2011$; accepted July $20^{\text {th }}, 2011$.

\begin{abstract}
Olmesartan Medoxomil (OLM), Ramipril (RPL) \& Fenofibric acid $(F A)$ are used to treat hypertension and cardiovascular disease. These drugs undergo hydrolytic metabolism by the enzyme liver esterase and converts into their respective active metabolites Olmesartan (OL), Ramiprilat (RPT) and Fenofibric acid (FA) for OLM, RPL and FEN respectively. In this study the competitive metabolism of OLM, in presence of RPL and FEN was investigated in rat liver S9 fractions using a validated LC-MS method. Olmesartan Medoxomil was found to be highly reactive to the rat liver S9 fractions and formation of active metabolite Olmesartan is highest. The rate of formation of active metabolite Olmesartan reduced by $12.68 \%$ in the presence Ramipril and $6.56 \%$ in presence of Fenofibrate. A marked reduction of $18.96 \%$ was found in the formation of active metabolite Olmesartan from Olmesartan Medoxomil when all the three drugs are in combination.
\end{abstract}

Keywords: In Vitro Metabolism, Omlesartan, Fenofibrate, Ramipril, Rat Liver, LC-MS

\section{Introduction}

Drug metabolism, basically an adaptive process, is a rather useful property of the (liver) cell, as a whole. Drug biotransformation usually leads to more polar compounds, and thus to faster elimination, and to substances with lower or no activity. Only rarely is an increase of activity observed after biotransformation. A common challenge during early development of a new drug candidate is to determine an analytical approach that is capable of delivering a versatile, cost effective and efficient analytical throughput in support of the preclinical program. The major steps in the in vitro drug metabolism studies are the identification and quantification of the drug and the metabolites.

Carrying out in vitro study is inexpensive and serves as an adequate screening mechanism that can rule out the importance of a metabolic pathway and make in vivo testing unnecessary. In vitro studies are able to help clinical study design to impact directly on the labelling of medicines, rationalise the contra-indications offered and consolidate the patient group in which dosing is expected to be safe and efficacious It may be concluded that the metabolism of drugs understudy is affected by other, hence combination/co-administration can be avoided.

It is common that a patient is taking several drugs for the treatment of one or more health related issues at the same time. A drug may affect or be affected by other co-administered drugs. Metabolism-based drug-drug interactions occur when a drug inhibits or induces the activity of a drug metabolizing enzyme, which catalyzes the metabolism of the concomitant drug. The metabolism-based drug-drug interaction is one of the major factors that cause drug failures during drug development. Early stage screening of compounds for potential drugdrug interactions using in vitro techniques becomes necessary in order to decrease late stage compound attrition. This early in vitro screening of drug-drug interactions facilitates the drug discovery and development process.

Olmesartan Medoxomil and Ramipril are antihypertensive drugs belongs to ACE inhibitors and $\mathrm{AT}^{1}$ antagonist category respectively. Fenofibrate is a co-administered drug with antihypertensives for its lipid lowering nature. All the three drugs are prodrugs and in liver, they undergo hydrolytic metabolism by the enzyme liver es- 
terase to form their active metabolites Olmesartan, Ramiprilat and Fenofibrate respectively, so there is a probability of competitive metabolism interactions of RPL and FA with OLM. A study was conducted to determine the effect of two drugs on the metabolism of OLM when available in combination or co administration. An extensive literature survey revealed that, number of analytical methods have been described for the estimation of Olmesartan Medoxomil [1-5], Ramipril [6-9], Fenofibrate [10-12] from various matrices like formulation and biological samples. No literatures are available for their metabolic interaction study and hence this paper describes an invitro interaction study between them using LC-MS method to predict the probability of interactions in their metabolic pathway. As there is no such method exists, this method would serve to analyze trace quantity of them.

\section{Materials and Methods}

\subsection{Incubation Conditions}

Rat liver S9 fractions (0.5 mg/mL, Lot. No. 029K1126), Sigma Aldrich Co. were used. Drug solutions were incubated for 60 mins with RLF in $2.0 \mathrm{ml}$ of incubation medium consisting of a tris-HCL buffer $(0.12 \mathrm{mM}, \mathrm{pH} 7.4$ at $\left.37^{\circ} \mathrm{C}\right), \mathrm{MgCl}_{2}(5 \mathrm{mM})$, Sodium pyrophosphate $(6.25 \mathrm{mM})$, D-glucose 6-phosphate $(5 \mathrm{mM})$, D-glucose 6-phosphate dehydrogenase $(1 \mathrm{U} / \mathrm{mL})$ and NADPH $(\beta$-Nicotinamide adenine dinucleotide 2-phosphate reduced tetrasodium salt, $0.5 \mathrm{mM}$ ). After $60 \mathrm{~min}$ of incubation $1 \mathrm{ml}$ of ice cooled methanol was added to stop the reaction by precipitation of proteins and the solution was centrifuged at $5000 \mathrm{rpm}$ for $5 \mathrm{~min}$ to separate out the proteins and the supernatant liquid was used for the study.

\subsection{LC-MS Conditions}

An end capped C18 reverse phase column (250 mm $\times 4.0$ $\mathrm{mm}, 5 \mu \mathrm{m})$, E. Merck was used for the study. Mobile phase consisted of acetonitrile, methanol and $0.35 \% \mathrm{v} / \mathrm{v}$ formic acid $(80: 20: 30 \mathrm{v} / \mathrm{v})$. The study was carried out at room temperature with a flow rate of $1.2 \mathrm{ml} / \mathrm{min}$ with positive polarity mode by keeping MS scan range 200 850 AMU. Drying gas flow was adjusted at $10 \mathrm{~L} / \mathrm{Hr}$ and temperature was fixed at $250^{\circ} \mathrm{C}$.

\subsection{LC-MS Analysis}

All the measurements were performed by LC-MS with ESI probe. The supernatants of the reaction mixtures were injected into an endcapped $\mathrm{C} 18$ reverse phase column $(250 \mathrm{~mm} \times 4.0 \mathrm{~mm}, 5 \mu \mathrm{m})$ and eluted at a flow rate of $1.2 \mathrm{ml} / \mathrm{min}$ in isocratic mode with a mobile phase consist of acetonitrile, methanol and $0.35 \% \mathrm{v} / \mathrm{v}$ formic acid $(80: 20: 30 \mathrm{v} / \mathrm{v})$. The mass peaks were observed respective to their $\mathrm{m} / \mathrm{z}$ values for the parent drugs and their active metabolites.

\subsection{Data Analysis}

The incubated solutions of individual drugs Olmesartan Medoxomil, Ramipril and Fenofibrate $(30 \mu \mathrm{M}$ of each drug) at different incubation time intervals of $0 \mathrm{~min}, 30$ min and 60 mins were injected in LC-MS system and the $\mathrm{M}+1$ ion peaks for the drugs and the metabolites were observed (OLM-559.35, OL-447.3, RPL-417.3, RPT389.2, FEN-361.2 and FA-319.1). Reduction in the drug peak intensities and increase in the metabolite peak intensities were observed carefully and relative intensities were calculated for all the three drug peaks and their metabolites. Relative percentage reduction of drugs peaks and increase in metabolite peaks were calculated using relative peak intensities for all the three drugs \& the metabolism pattern was studied using a plot with time on $\mathrm{X}$ axis and relative percentage increase in active metabolite peak on $\mathrm{Y}$ axis. Peak intensities of drugs and their expected metabolites were noted with single drug, as well as in combinations. The effect of each drug on other drug's metabolism was calculated based on relative peak intensity.

\section{Results and Discussion}

The biotransformation of Olmesartan Medoxomil to active metabolite Olmesartan, ramipril to ramiprilat and fenofibrate to fenofibric acid by the enzyme liver esterase present in S9 fraction are shown in Figures 1-3. An accurate and sensitive LC-MS method was developed and applied for carrying out in vitro metabolism interaction study, which is a screening mechanism in drug discovery. The drugs and metabolites were studied by MS using their respective $M+1$ value. Based on the peak intensity of drug and expected metabolite, the metabolism was studied. The mass spectrum of three drugs and their metabolites are represented in Figures 4-6.

The percentage formation of active metabolite Olmesartan from Olmesartan Medoxomil in the presence of Ramipril and Fenofibrate was compared with Olmesartan Medoxomil alone (Table 1). Similarly for all drugs the study was done and it was conducted for six times on every occasion. The rate of formation of active metabolite Olmesartan is highest among all. The rate of formation of active metabolite Olmesartan reduced by $12.68 \%$ in the presence Ramipril and $6.56 \%$ in presence of Fenofibrate. A marked reduction of $18.96 \%$ was found in the formation of active metabolite Olmesartan from Olmesartan Medoxomil when all the three drugs were in combination. It may be concluded from the study that the metabolism of OLM is affected by the presence of other 


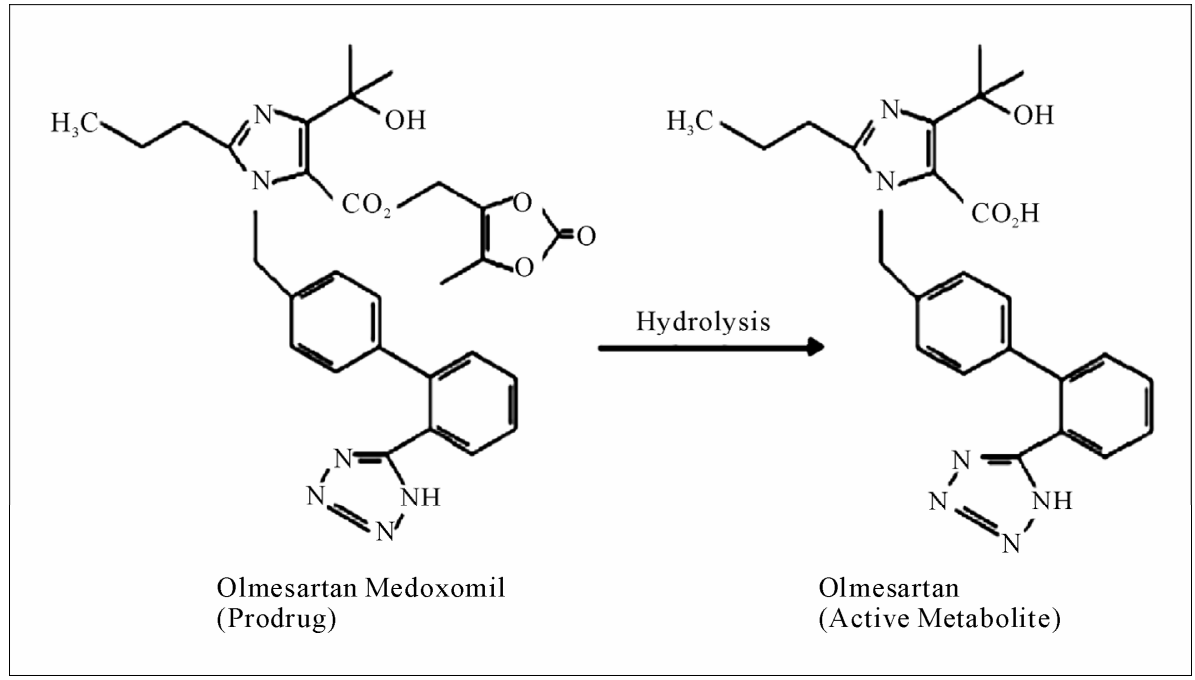

Figure 1. Metabolism of Olmesartan Medoxomil.

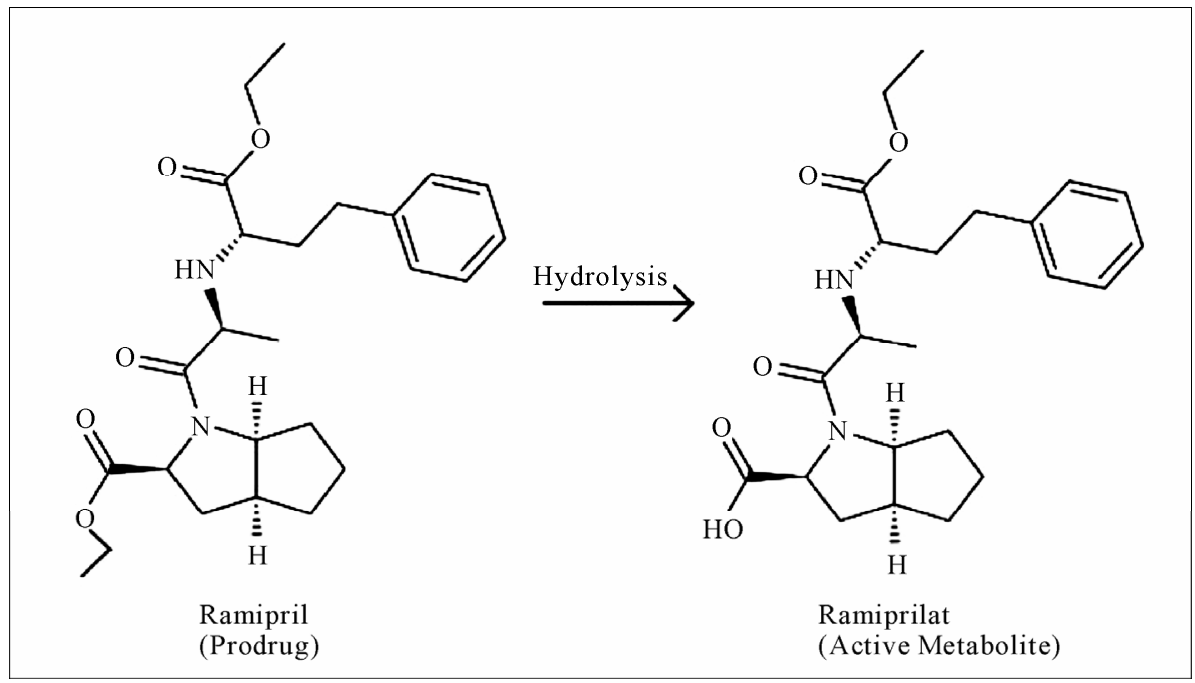

Figure 2. Metabolism of Ramipril.

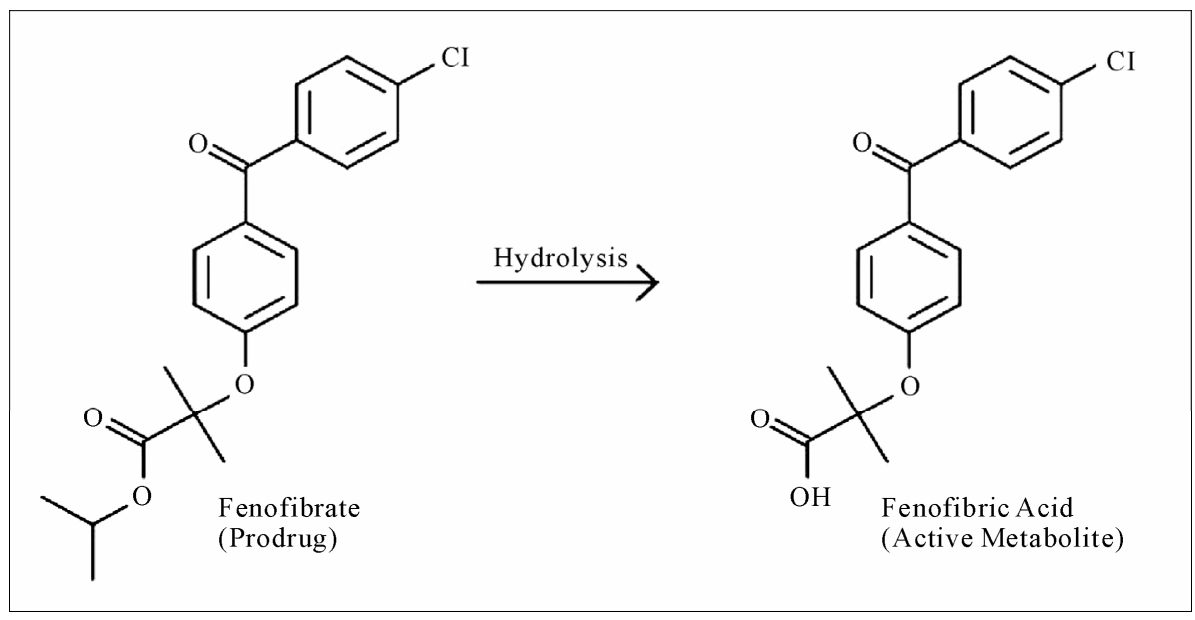

Figure 3. Metabolism of Fenofibrate. 


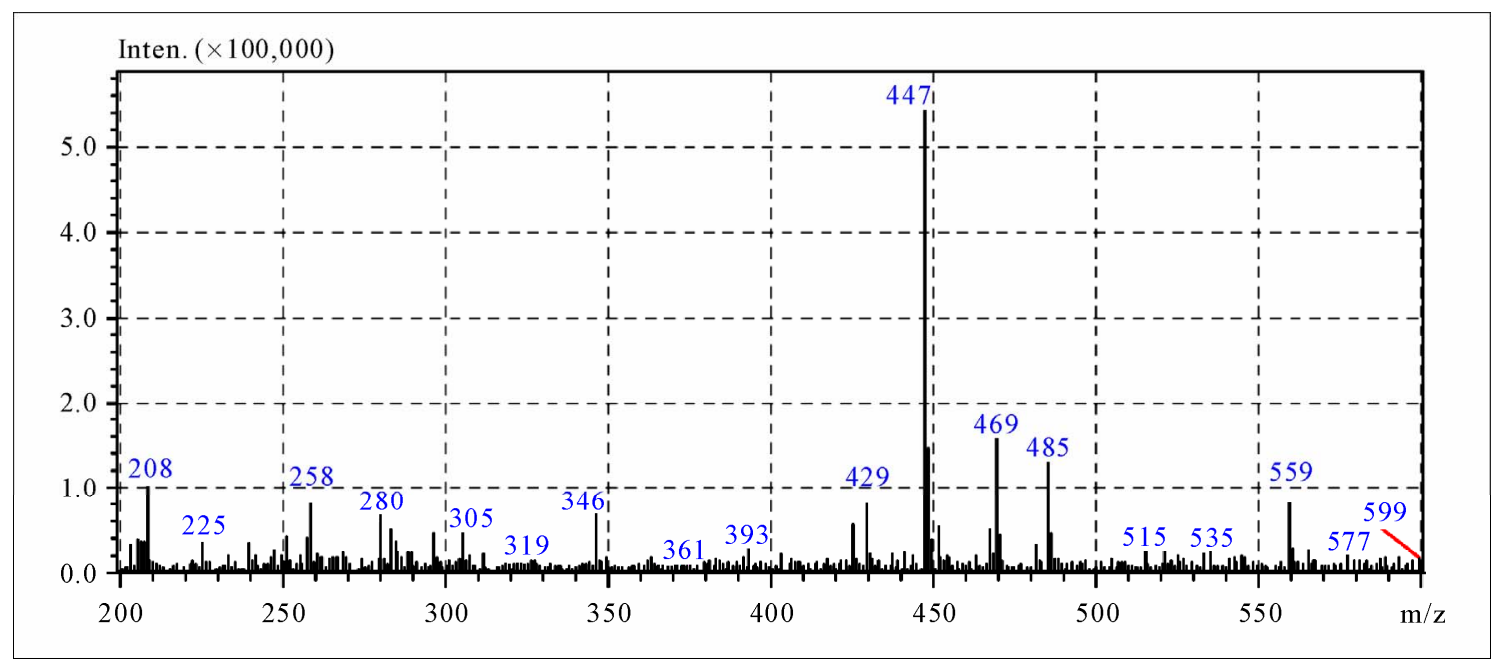

Figure 4. Mass spectra of Olmesartan Medoximil (447) and Olmesartan (429).

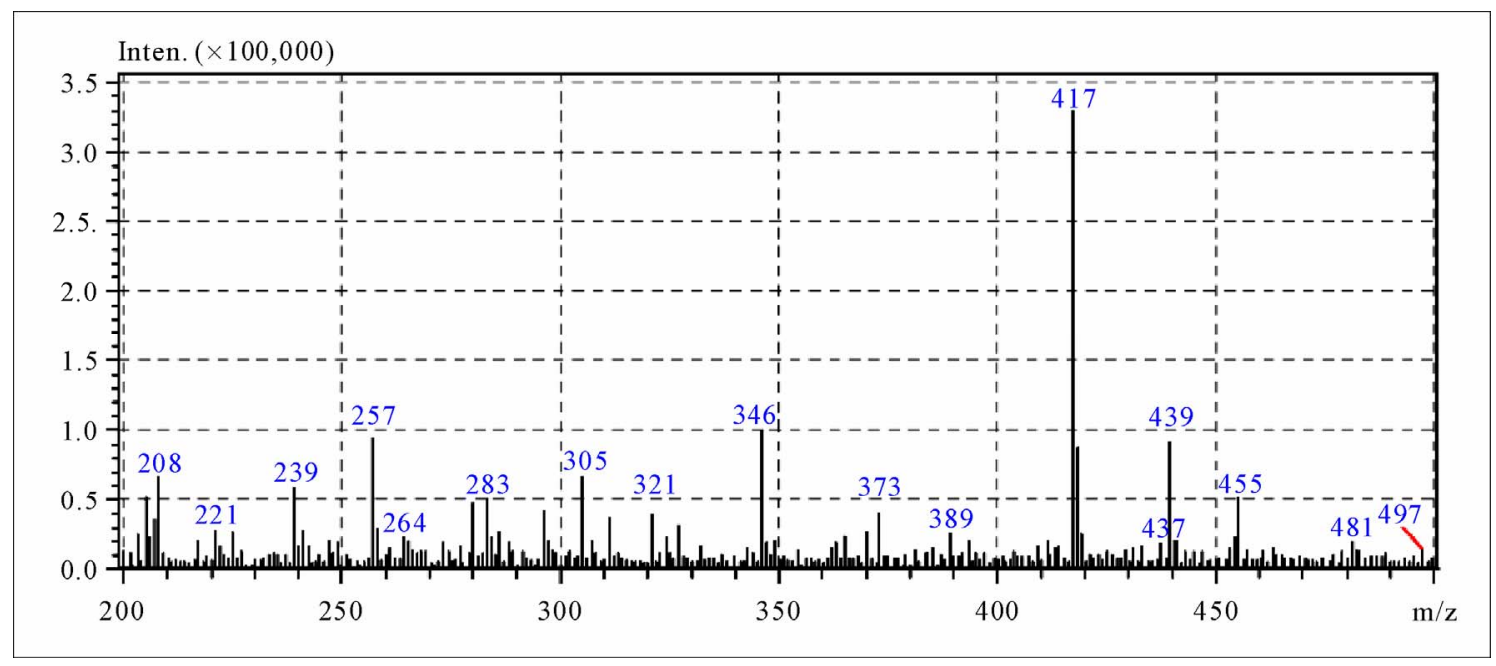

Figure 5. Mass spectrum of Ramipril (417) and Ramiprilat (389).

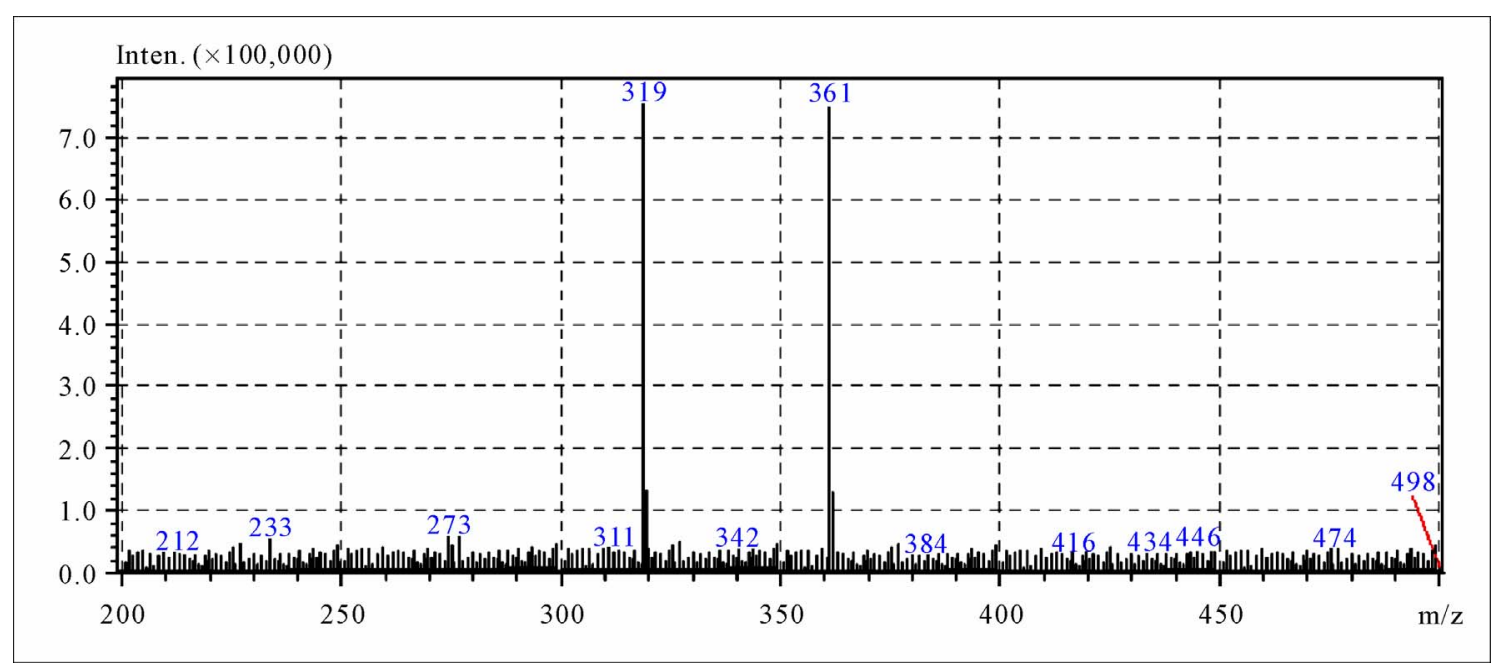

Figure 6. Mass spectrum of Fenofibrate (361) and finofibric acid (319). 
Table 1. Relative percentage Olmesartan formation.

\begin{tabular}{lcccccc}
\hline \multirow{2}{*}{ Drug } & \multicolumn{6}{c}{ Relative Percentage of Olmesartan Formation } \\
\cline { 2 - 7 } & $0 \mathrm{~min}$ & $\% \mathrm{RSD}$ & $30 \mathrm{~min}$ & $\%$ RSD & $60 \mathrm{~min}$ & \%RSD \\
\hline OLM & 5.61 & 4.200 & 67.44 & 5.241 & 95.04 & 4.391 \\
OLM + FEN & 6.39 & 3.547 & 63.96 & 3.652 & 88.48 & 5.330 \\
OLM + RPL & 10.67 & 2.846 & 65.38 & 2.845 & 82.36 & 4.788 \\
OLM + RPL + FEN & 7.55 & 4.529 & 59.42 & 3.139 & 76.08 & 4.221 \\
\hline
\end{tabular}

drugs; hence combination or co-administration of such drugs could be avoided.

\section{REFERENCES}

[1] N. Sultana, M. S. Arayane, S. S Ali and S. Sajid, "Simultaneous Determination of Olmesartan Medoxomil and Irbesartan and Hydrochlorothiazide in Pharmaceutical Formulations and Human Serum Using High Performance Liquid Chromatography," Chinese Journal of Chromatography, Vol. 26, No. 5, 2008, pp. 544-549. doi:10.1016/S1872-2059(08)60029-2

[2] B. Lisiane, R. C. Rochele, D. L. Carolina B. M. Ana and F. E. Pedro, "Stability-Indicating LC Determination of a New Antihypertensive, Olmesartan Medoxomil in Tablets," Chromatographia, Vol. 68, No. 11-12, 2008, pp. 991-996. doi:10.1365/s10337-008-0811-3

[3] P. D. Bari and A. Rote, "RP-LC and HPTLC Methods for the Determination of Olmesartan Medoxomil and Hydrochlorothiazide in Combined Tablet Dosage Forms," Chromatographia, Vol. 69, No. 11-12, 2009, pp. 1469-1472. doi:10.1365/s10337-009-1094-Z

[4] S. G. Chrysant, "Amlodipine Besylate/Olmesartan Medoximil Fixed Combination for the Treatment of Hypertension," Expert Review of Cardiovascular Therapy, Vol. 7, No. 8, 2009, pp. 887-895. doi:10.1586/erc.09.85

[5] D. Liua, P. Hu, N. Matsushima, X. Lia, L. Lia and J. Ji, "Quantitative Determination of Olmesartan in Human Plasma and Urine by liquid Chromatography Coupled to Tandem Mass Spectrometry," Journal of Chromatography B, Vol. 856, No. 1-2, 2007, pp. 190-197. doi:10.1016/j.jchromb.2007.05.049

[6] B. Yuan, X. Wang, F. Zhang, J. Jia and F. Tang, "Simultaneous Determination of Ramipril and Its Active Metabolite Ramiprilat in Human Plasma by LC-MS-MS," Chromatographia, Vol. 68, No. 7-8, 2008, pp. 533-539. doi:10.1365/s10337-008-0757-5
[7] B.-A. Persson, C. Fakt, M. Ervik and M. Ahnoff, "Interference from a Glucuronide Metabolite in the Determination of Ramipril and Ramiprilat in Human Plasma and Urine by Gas Chromatography-Mass Spectrometry," Journal of Pharmaceutical and Biomedical Analysis, Vol. 40, No. 3, 2006, pp. 794-797.

[8] X.-Y. Lu, J.-Z. Shen-Tu and J. Liu, "High-Performance Liquid Chromatography-Mass Spectrometric Analysis of Ramipril and Its Active Metabolite Ramiprilat in Human Serum: Application to a Pharmacokinetic Study in the Chinese Volunteers," Journal of Pharmaceutical and Biomedical Analysis, Vol. 40, No. 2, 2006, pp. 478-483. doi:10.1016/j.jpba.2005.07.054

[9] Z. Zhimeng, "Liquid Chromatography-Mass Spectrometry Method for Determination of Ramipril and Its Active Metabolite Ramiprilat in Human Plasma," Journal of Chromatography B, Vol. 779, No. 2, 2002, pp. 297-306. doi:10.1016/S1570-0232(02)00398-7

[10] G. E. Linda, "Evaluation of the Potential for Pharmacokinetic Interaction between Fenofibrate and Ezetimibe: A Phase I, Open-Label, Multiple-Dose, Three-Period Crossover Study in Healthy Subjects," Clinical Therapeutics, Vol. 28, No. 3, 2006, pp. 373-387. doi:10.1016/j.clinthera.2006.03.009

[11] Z. Zhu, A. Vachareau and L. Neirinck, "Liquid Chromatography-Mass Spectrometry Method for Determination of Ramipril and Its Active Metabolite Ramiprilat in $\mathrm{Hu}$ man Plasma," Journal of Chromatography B, Vol. 779, No. 2, 2002, pp. 297-306. doi:10.1016/S1570-0232(02)00398-7

[12] V. P. Chintan, P. K. Amit, D. C. Anandi and T. P. Kalpesh, "Validated Absorption Factor Spectrophotometric and Reversed-Phase High-Performance Liquid Chromatographic Methods for the Determination of Ramipril and Olmesartan Medoxomil in Pharmaceutical Formulations," Eurasian Journal of Analytical Chemistry, Vol. 2, No. 3, 2007, pp. 159-171. 